\title{
Covid-19 e burnout em enfermeiros residentes de um hospital universitário
}

\author{
Covid-19 and burnout in resident nurses at a university hospital \\ Covid-19 y burnout en enfermeros residentes de un hospital universitario
}

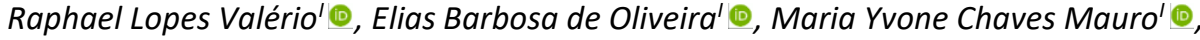 \\ Regina Célia Gollner Zeitoune" @
}

'Universidade do Estado do Rio de Janeiro, Rio de Janeiro, RJ, Brasil; "Universidade Federal do Rio de Janeiro, Rio de Janeiro, RJ, Brasil

\section{RESUMO}

Objetivo: analisar a ocorrência de burnout em enfermeiros residentes de unidades Covid-19 de um hospital universitário. Método: quantitativo, descritivo do tipo transversal com uma amostra de 40 enfermeiros residentes de um hospital universitário público situado no município do Rio de Janeiro. A coleta de dados foi realizada de outubro a dezembro de 2020 , via Google Forms, mediante instrumento de caracterização da amostra e o Maslach Burnout Inventory - Human Services Survey. Resultados: prevaleceram residentes do sexo feminino, casados e maiores de 25 anos. Verificou-se que $12,5 \%$ da amostra preencheram os critérios para burnout com risco de desenvolvimento da síndrome devido a altos escores em exaustão emocional (55\%), médios em despersonalização (47,5\%) e baixa realização profissional (20\%). Conclusão: a pandemia aumentou os riscos de burnout na amostra, tornando-se necessário investimentos em suporte social e técnico por parte das instituições formadoras de modo a minimizar o adoecimento.

Descritores: Enfermagem; Infecções por Coronavírus; Educação de Pós-Graduação; Esgotamento Profissional; Saúde Mental.

\section{ABSTRACT}

Objective: to analyze the occurrence of burnout among resident nurses at Covid-19 units of a university hospital. Method: in this quantitative, descriptive, cross-sectional study of a sample of 40 nurses residing in a public university hospital in Rio de Janeiro city, data were collected from October to December 2020 on Google Forms, using an instrument to characterize the sample and the Maslach Burnout Inventory - Human Services Survey. Results: the residents were predominantly female, married and over 25 years old. Scores for emotional exhaustion were high (55\%), for depersonalization, average (47.5\%) and for professional achievement, low (20\%), and $12.5 \%$ of the sample met the criteria for burnout with risk of developing the syndrome. Conclusion: the pandemic increased burnout risks in the sample, requiring educational institutions to invest more in social and technical support to minimize illness.

Descriptors: Nursing; Coronavirus Infections; Graduate Education; Professional Burnout; Mental Health.

\section{RESUMEN}

Objetivo: analizar la ocurrencia de burnout en enfermeras residentes en unidades Covid-19 de un hospital universitario. Método: cuantitativo, descriptivo del tipo transversal con una muestra de 40 enfermeros residentes de un hospital universitario público ubicado en la ciudad de Río de Janeiro. La recolección de datos se realizó de octubre a diciembre de 2020, vía Google Forms, utilizando un instrumento para caracterizar la muestra y el Maslach Burnout Inventory - Human Services Survey. Resultados: predominaron residentes del sexo femenino, casadas y mayores de 25 años. Se encontró que el $12,5 \%$ de la muestra cumplía con los criterios de burnout con riesgo de desarrollar el síndrome debido a puntuaciones altas en agotamiento emocional (55\%), medianas en despersonalización $(47,5 \%$ y bajas en realización profesional (20\%). Conclusión: la pandemia aumentó los riesgos de burnout en la muestra, volviendo necesario realizar inversiones en apoyo social y técnico por parte de las instituciones educativas para minimizar la enfermedad Descriptores: Enfermería; Infecciones por Coronavirus; Educación de Postgrado; Agotamiento Profesional; Salud Mental.

\section{INTRODUÇÃO}

Em março de 2020, a Organização Mundial de Saúde (OMS) caracterizou como estado de pandemia a doença causada pelo Severe Acute Respiratory Syndrome Coronavirus 2 (SARS-Cov-2) ou Covid-19. Na segunda semana de julho de 2021 foram confirmadas 187.296.646 notificações da doença e 4.046.470 mortes no mundo. O Brasil ocupa o terceiro lugar com o maior número de registros da infecção e, também, em número de mortes, envolvendo inclusive trabalhadores da saúde. Com a vacinação em massa, tem-se observado uma tendência de diminuição do número de casos e mortes. No entanto, com o surgimento de infecções entre pessoas vacinadas e as novas cepas do vírus, há necessidade de se manter medidas protetivas, tais como: restrição social, lavagem das mãos e o uso de máscara pela população dentre outras ${ }^{1,2}$.

A pandemia alterou a rotina dos serviços e impôs aos gestores e profissionais de saúde a urgência de adaptação ao novo cenário, acarretando intensificação das cargas física e psíquica do trabalho com implicações para a saúde dos trabalhadores,

Autor Correspondente: Raphael Lopes Valério. E-mail: raphael_rlv@hotmail.com

Editora Científica: Cristiane Helena Gallasch; Editora Associada: Magda Guimarães de Araujo Faria 
diante do risco de exposição ao vírus, adoecimento e morte ${ }^{3}$. Tais cargas remetem a aspectos relacionados ao cuidado dos pacientes e outras demandas envolvendo a organização do trabalho hospitalar devido ao déficit de recursos humanos, de treinamento, de orientações claras sobre o tratamento e disponibilidade de equipamentos de proteção individual e coletiva ${ }^{4,5}$.

Os trabalhadores que atuam nas unidades de atendimento de Covid-19, têm sido um dos grupos mais afetados pela pandemia, em que se observa quadros de sofrimento psíquico acompanhados de sintomatologia de estresse, ansiedade e reações depressivas ${ }^{6-9}$. Entre os fatores de risco para o desenvolvimento da Síndrome de Burnout em profissionais da saúde durante a pandemia cabe destacar a sobrecarga de trabalho, fadiga, estresse, depressão e interação social comprometida ${ }^{10}$.

Os residentes como parte da equipe de saúde, por serem em sua maioria jovens, inexperientes e possuírem estratégias adaptativas pouco responsivas no enfrentamento dos estressores psicossociais do trabalho, encontram-se mais propensos ao risco de desenvolvimento da Síndrome de Burnout, tendo a qualidade de vida afetada nas dimensões física e psicossocial ${ }^{11,12}$. Além das características individuais, estressores relacionados à estruturação do curso podem contribuir para a exaustão emocional e entre eles: o alto grau de responsabilidade, a ausência dos tutores nos campos de prática, a carga horária extensa, as atividades teóricas, o trabalho em turnos, inclusive noturno e nos finais de semana, a reduzida autonomia e as relações de poder institucionalizadas ${ }^{13-15}$.

O pouco conhecimento acumulado pela ciência acerca do vírus e da própria doença durante a pandemia tem criado dificuldades para o seu enfrentamento em termos de terapêuticas e/ou tratamento adotados pelas instituições de saúde frente o adoecimento da população e o risco de morte. Nesse sentido, experiências exitosas voltadas para a prevenção, promoção da saúde e tratamento das desordens físicas e/ou psíquicas relatadas por outros países, têm sido absorvidas e/ou adaptadas à nossa realidade². Tais movimentos vão ao encontro da campanha iniciada pelo Ministério da Saúde (MS) e pela Organização Pan-Americana da Saúde (OPAS) ao reconhecer a relevância de estratégias dirigidas à mitigação do estresse e angústia dos profissionais de saúde no contexto da pandemia ${ }^{16}$.

A Síndrome de Burnout é uma resposta do organismo a um processo progressivo de exaustão emocional e perda do interesse do profissional quando os métodos de enfrentamento falham ou são insuficientes. É mais frequente nos trabalhadores que exercem atividades de cuidado, envolvendo três fatores multidimensionais propostos a partir do Malasch Burnout Inventory: exaustão emocional caracterizada por baixo entusiasmo e sensação de esgotamento de recursos; despersonalização ou insensibilidade marcada por atitudes negativas de distanciamento, intolerância e tratamento impessoal de pacientes e equipe; e reduzida realização profissional na qual há sensação de insuficiência e baixa autoestima ${ }^{17}$.

No desenvolvimento da síndrome de Burnout a pessoa pode apresentar queixas de ordem física (dores musculares, fadiga constante, distúrbios gastrintestinais e neuroendócrinos), psíquicas (alterações da memória, da concentração, lentidão e ruminação de pensamentos), emocionais (ansiedade, irritabilidade, depressão e agressividade) e comportamentais (isolamento, negligência, falta de interesse pelo trabalho ou lazer, inflexibilidade). Essas alterações podem acarretar desinteresse pelas atividades do dia a dia e trabalho, sendo observado, por vezes, o consumo de substâncias psicoativas como estratégias de minimização do sofrimento ${ }^{18}$. Indivíduos perfeccionistas, competitivos, impacientes, controladores, com dificuldade de tolerar frustração e que atribuem grande significado ao trabalho, têm maior propensão ao desenvolvimento da síndrome ${ }^{19}$.

Como os problemas de saúde mental tendem a se agravar e/ou se ampliar durante a pandemia ${ }^{9,10,16}$, o presente estudo se justifica por ratificar o papel das instituições de ensino e saúde na prevenção do estresse psicossocial e acolhimento dos residentes de enfermagem, contribuindo para o processo de formação, promoção da saúde e qualidade de vida. Diante do exposto, o presente estudo teve como objetivos analisar a ocorrência de burnout em enfermeiros residentes de unidades Covid-19 de um hospital universitário.

\section{MÉTODO}

Estudo quantitativo, transversal, descritivo. O campo foi um hospital universitário público de grande porte, situado no município do Rio de Janeiro que possui unidades de atendimento à Covid-19. Trata-se de uma unidade reconhecida por sua excelência nas áreas de pesquisa, assistência, extensão e formação de profissionais nos níveis de graduação e pós-graduação, incluindo programas de residência em várias áreas.

A residência de enfermagem da instituição é estruturada nos preceitos legais da Resolução 657/2020 do Conselho Federal de Enfermagem ${ }^{20}$, com duração de quatro semestres letivos e organizada em três áreas e respectivos programas: enfermagem clínica (clínica médica, terapia intensiva, nefrologia, saúde do adolescente, psiquiatria e saúde do trabalhador); enfermagem cirúrgica (centro cirúrgico, clínica cirúrgica e cardiovascular); materno infantil (neonatologia, obstetrícia e pediatria). 
De uma população de 87 residentes, participaram do estudo 40 residentes de enfermagem do primeiro e segundo anos, mediante amostragem não probabilística ou por conveniência, tendo os seguintes critérios de inclusão: residentes matriculados no curso e que estivessem realizando as atividades técnico-assistenciais em unidades de Covid-19, sendo excluídos os residentes de férias e afastados para tratamento médico ou de outra natureza. Devido ao risco de exposição ao Covid-19 por parte dos pesquisadores, optou-se pela coleta de dados, ocorrida no período de outubro a dezembro de 2020, por meio da plataforma digital Google Forms. Os endereços eletrônicos dos participantes foram obtidos junto a chefia do serviço de treinamento e avaliação de enfermagem da instituição.

Após acesso ao formulário online contendo o convite, leitura dos dados sobre o estudo e informações acerca dos objetivos e aspectos éticos da pesquisa, todos assinalaram a caixa de diálogo concordando com o termo de consentimento livre e esclarecido. Ratificou-se no instrumento que a participação seria voluntária e garantiu-se o direito de o residente retirar o consentimento em qualquer fase do estudo. Após assinalar a concordância dos aspectos expresso no termo, uma via do consentimento foi encaminhada automaticamente para o participante e a outra anexada no banco de dados.

Na coleta de dados, utilizou-se um instrumento estruturado elaborado pelos autores contendo dez itens sobre as variáveis de exposição sociodemográficas (sexo, faixa etária, estado civil), formação (ano da residência, tempo de formado e experiência profissional) e condições de saúde (licença médica nos últimos doze meses, afastamento devido a Covid-19, acompanhamento em saúde mental e consumo de bebidas alcoólicas). Na suspeição da Síndrome de Burnout, optou-se pelo inventário Maslach Burnout Inventory (MBI) versão Human Services Survey (MBI-HSS), elaborado por Maslach, Leiter e Jackson ${ }^{17}$.

$\mathrm{O}$ instrumento foi traduzido e validado no país por Lautert ${ }^{19}$ em estudo realizado com enfermeiras da área hospitalar. Possui 22 itens sob a forma de afirmações cujos escores são estimados mediante uma escala do tipo Likert com opções de respostas de nunca (0) a todos os dias (5) que avaliam as três dimensões da Síndrome de Burnout: nove itens em Exaustão Emocional (EE), cinco em Despersonalização (DP) e oito em Realização Profissional (RP).

No intuito de identificar a suspeição da Síndrome de Burnout na amostra, trabalhou-se inicialmente com as respostas de MBI-HSS de cada participante, partindo do pressuposto que na suspeição da Síndrome de Burnout, há necessidade de altos escores nas subescalas EE, DP e baixo escore em $\mathrm{RP}^{17}$, a partir dos seguintes pontos de corte: $\mathrm{EE}$ - pontuações maiores de 21 (Alta EE); de 11 a 21 (Média EE); valores menores ou igual a 10 (Baixa EE); DP - pontuações maiores de 8 (Alta DP); de 3 a 8 (Média DP); e menor ou igual a 2 (Baixa DP). A RP apresenta medida reversa, em que pontuação igual a 27 ou acima (Alta RP) não condiz com a Síndrome de Burnout; de 21 a 26 (Média RP) e igual ou menor de 20 (Baixa RP) ${ }^{17,18}$.

$\mathrm{Na}$ descrição das variáveis de exposição, optou-se pela estatística descritiva (frequências absoluta e relativa), sendo os resultados discutidos com base, principalmente nos estudos realizados sobre a Síndrome de Burnout em residentes e profissionais da saúde e de enfermagem.

O estudo tem por base a resolução 466/2012 ${ }^{21}$, do Conselho Nacional em Saúde, que trata sobre a realização de pesquisas envolvendo seres humanos. Foi aprovado pelo Comitê de Ética em Pesquisa da instituição, sob o parecer $n$ 은 4.109.547.

\section{RESULTADOS E DISCUSSÃO}

Identificou-se na amostra cinco participantes (12,5\%) que preencheram todos os requisitos de suspeição de burnout, ao considerar as subescalas e respectivos pontos de corte. Quanto à análise das dimensões envolvidas optouse pela descrição coletiva das respostas ao instrumento mediante estatística descritiva (Tabela 1).

TABELA 1: Distribuição dos escores das subescalas da Síndrome de Burnout na amostra de acordo com o MBI-HSS ( $n=40)$. Rio de Janeiro, RJ, Brasil, 2021.

\begin{tabular}{lccc}
\hline Exaustão emocional & Escores & $\mathbf{n}$ & $\%$ \\
\hline Alta & $>21$ & 22 & 55 \\
Média & 11 a 21 & 13 & 32,5 \\
Baixa & $<$ ou $=10$ & 05 & 12,5 \\
Despersonalização & & & \\
Alta & $>8$ & 14 & 35 \\
Média & 03 a 08 & 19 & 47,5 \\
Baixa & $<$ ou $=02$ & 07 & 17,5 \\
Realização Profissional & & & \\
Baixa & $<$ ou $=20$ & 08 & 20 \\
Média & 21 a 27 & 09 & 22,5 \\
Alta & $>$ ou $=$ a 27 & 23 & 57,5 \\
\hline
\end{tabular}

Fonte: Os autores, 2020 
Como constatado, uma parcela expressiva dos residentes apresentou risco de desenvolvimento da Síndrome de Burnout diante dos altos escores nas subescalas EE (55\%), DP (35\%) e baixos para RP (20\%).

A presença de alta EE é sugestiva de exposição do indivíduo a estressores ambientais e cujos recursos adaptativos em termos de respostas fisiológicas, psicológicas e comportamentais podem não ser eficazes na sua minimização ou eliminação. Há um esforço cognitivo e comportamental da pessoa no intuito de atuar na própria emoção e/ou pensamentos, mudar a sua perspectiva ou trabalhar a situação geradora do estresse de modo a torná-la condizente com suas expectativas e com o menor gasto de energia. Daí a importância das estratégias de coping adotadas, que dependerão do repertório pessoal e profissional em termos de vivências e experiências relacionadas ao contexto de trabalho 22 .

De acordo com a Tabela 2, a amostra foi composta majoritariamente por residentes do sexo feminino, casadas ou em união consensual, faixa etária acima de 25 anos, com dois ou mais anos de formados, cursando o segundo ano da residência e sem experiência profissional. Uma parcela significativa de residentes foi afastada do curso para tratamento de problemas de saúde nos últimos doze meses que antecederam a coleta, inclusive devido a Covid-19. Verificou-se também o acompanhamento de residentes pela saúde mental e o consumo de bebidas alcóolicas.

Tabela 2: Distribuição dos residentes de enfermagem segundo as variáveis de exposição e subescalas do MBI-HSS (n=40). Rio de Janeiro. Brasil, 2020.

\begin{tabular}{|c|c|c|c|c|c|c|c|c|}
\hline \multirow{3}{*}{ Variáveis } & & & \multicolumn{6}{|c|}{ Dimensões do Burnout } \\
\hline & \multicolumn{2}{|c|}{ Amostra } & \multicolumn{2}{|c|}{$E E(n=22)$} & \multicolumn{2}{|c|}{$\mathrm{DP}(\mathrm{n}=14)$} & \multicolumn{2}{|c|}{$\mathrm{RP}(\mathrm{n}=8)$} \\
\hline & $\mathrm{n}$ & $\%$ & $\mathbf{n}$ & $\%$ & $\mathbf{n}$ & $\%$ & $\mathbf{n}$ & $\%$ \\
\hline \multicolumn{9}{|l|}{ Sexo } \\
\hline Feminino & 35 & 87,5 & 20 & 90,9 & 12 & 85,7 & 08 & 100 \\
\hline Masculino & 05 & 12,5 & 02 & 9,1 & 02 & 14,3 & - & - \\
\hline \multicolumn{9}{|c|}{ Vive com companheiro(a) } \\
\hline Sim & 27 & 67,5 & 13 & 59,1 & 09 & 64,3 & 07 & 87,5 \\
\hline Não & 13 & 32,5 & 09 & 40,9 & 05 & 35,7 & 01 & 12,5 \\
\hline \multicolumn{9}{|l|}{ Faixa etária } \\
\hline Até 25 anos & 16 & 40 & 10 & 45,5 & 05 & 35,7 & 03 & 37,5 \\
\hline Acima de 25 anos & 24 & 60 & 12 & 54,5 & 09 & 64,3 & 05 & 62,5 \\
\hline \multicolumn{9}{|l|}{ Tempo de Formado } \\
\hline 1 ano & 12 & 30 & 07 & 31,8 & 02 & 14,3 & 01 & 12,5 \\
\hline 2 ou $>$ anos & 28 & 70 & 15 & 68,2 & 12 & 85,7 & 07 & 87,5 \\
\hline \multicolumn{9}{|c|}{ Experiência profissional } \\
\hline Sim & 09 & 22,5 & 07 & 31,8 & 06 & 42,8 & 01 & 12,5 \\
\hline Não & 31 & 77,5 & 15 & 68,2 & 08 & 57,2 & 07 & 87.5 \\
\hline \multicolumn{9}{|c|}{ Turma de Residente } \\
\hline Primeiro ano & 15 & 37,5 & 07 & 31,8 & 04 & 28,6 & 02 & 25 \\
\hline Segundo ano & 25 & 62,5 & 15 & 68,2 & 10 & 71,4 & 06 & 75 \\
\hline \multicolumn{9}{|l|}{ Licença médica } \\
\hline Sim & 25 & 62,5 & 12 & 54,5 & 08 & 57,2 & 06 & 75 \\
\hline Não & 15 & 37,5 & 10 & 45,5 & 06 & 42,8 & 02 & 25 \\
\hline \multicolumn{9}{|c|}{ Licença devido a Covid-19 } \\
\hline Sim & 24 & 60 & 11 & 50 & 07 & 50 & 06 & 75 \\
\hline Não & 16 & 40 & 11 & 50 & 07 & 50 & 02 & 25 \\
\hline \multicolumn{9}{|c|}{ Acompanhamento em SM } \\
\hline Sim & 13 & 32,5 & 08 & 36,4 & 04 & 28,6 & 03 & 37,5 \\
\hline Não & 27 & 67,5 & 14 & 63,6 & 10 & 71,4 & 05 & 62,5 \\
\hline \multicolumn{9}{|c|}{ Uso de bebidas alcóolicas } \\
\hline Sim & 21 & 52,5 & 10 & 45,5 & 10 & 71,4 & 08 & 100 \\
\hline Não & 19 & 47,5 & 12 & 54,5 & 04 & 28,6 & - & - \\
\hline
\end{tabular}

Fonte: Os autores. Nota: SM: Saúde Mental. 2020.

Alguns dados sociodemográficos, como sexo feminino, estado civil casado e ter idade superior a 25 anos apresentaram relação com o risco de desenvolvimento da Síndrome de Burnout na amostra, considerando altos escores em EE, DP e baixa RP. Estudo sobre Burnout em residentes médicos e a associação com as características sociodemográficas apresentou diferença estatisticamente significativa para o desenvolvimento da síndrome em mulheres e despersonalização nos homens. Por sua vez, a existência de poucos estudos sobre burnout e gênero é um dado de interpretação bastante limitado ${ }^{14}$. 
Quanto ao estado civil, uma parcela significativa de residentes casados ou em união consensual apresentou altos escores em EE, DP e baixo senso de RP. Esses dados vão ao encontro de estudo com residentes de enfermagem cuja variável estado civil casada foi associada ao estresse na fase de resistência ao final do curso, sendo a qualidade de vida afetada nos domínios vitalidade com predomínio de sintomas psicológicos ${ }^{23}$.

Sobre a faixa etária, houve pouca diferença estatística entre os extremos e/ou amplitude, refletindo um perfil de enfermeiros recém-formados, egressos dos cursos de graduação e sem experiência profissional. Pelo fato de o estudo ter sido realizado com residentes do primeiro ano (R1) e segundo (R2), ao se analisar o risco da Síndrome de Burnout no grupo, deve-se considerar as diferenças em termos de experiências e/ou habilidades no enfrentamento dos estressores presentes no ambiente formador.

No presente estudo, a maior parte dos residentes foi convocada a trabalhar durante a pandemia nas unidades de Covid-19. Nessa situação, os R2 podem se sentir sobrecarregados diante do senso de responsabilidade e por responderem, em algumas situações, pela gerência do serviço, supervisão de graduandos e residentes do primeiro ano. Tais fatores podem explicar, em parte, o risco de burnout diante de altos escores em EE, DP e baixo senso de RP em comparação com o R1. Contrariando esses dados, estudo que avaliou a Síndrome de Burnout em residentes de um programa de residência multiprofissional, evidenciou uma porcentagem expressiva de residentes do primeiro ano (R1) em situação de risco para o desenvolvimento da Síndrome de Burnout, considerando altos escores em EE e RP baixa. Fatores como inexperiência, insegurança, cobranças por parte de pacientes, familiares e equipe foram os principais fatores associados ao desfecho ${ }^{11}$

A EE é considerada o traço inicial da Síndrome de burnout, devendo-se atentar para a susceptibilidade individual e aos mecanismos adaptativos diante dos estressores psicossociais ${ }^{17,18}$. Por outro lado, alguns aspectos organizacionais podem favorecer o estresse psicossocial em residentes, pois com a pandemia os hospitais passaram por mudanças estruturais e protocolares que afetaram as instituições de saúde, o processo de trabalho e a oferta de serviços em quantidade e qualidade. Houve necessidade de abertura de novas unidades de tratamento, ampliação dos leitos de terapia intensiva e urgência de treinamento das novas equipes de trabalho ${ }^{2,4,5}$

Apesar de os conhecimentos e habilidades adquiridas durante a graduação e a residência serem imprescindíveis para a atuação dos residentes nos campos de prática, cuidar de pacientes acometidos pela Covid-19, constitui-se em um grande desafio para o enfermeiro residente diante de problemas como: ausência de terapias eficazes em termos de tratamento, complicações e morte dos pacientes, manuseio de tecnologias de ponta e infecção ${ }^{16}$. Esse contexto de trabalho mostra-se propício à intensificação dos problemas de saúde mental em residentes como depressão, burnout e transtornos mentais comuns que já eram discutidos em estudos anteriores a pandemia ${ }^{12-14}$. Acrescenta-se os fatores estressores da formação envolvendo a dedicação ao curso, o cumprimento de uma carga horária extensa e o desgaste diante da necessidade de acumular vínculo empregatício como forma de complementação da renda ${ }^{15}$.

Apesar de os residentes recorrerem a estratégias de enfrentamento (coping) individual e coletiva no intuito de se adaptarem aos estressores presentes no ambiente de formação, tais estratégias, dependendo das características do indivíduo e das demandas organizacionais, podem não ser eficazes na prevenção da EE e DP ${ }^{22,23}$. A pandemia, por sua vez, contribui para o aumento de esforços físicos, cognitivos e afetivos em decorrência dos estressores psicossociais relacionados à complexidade das atividades e menor controle do processo de trabalho. Os profissionais que atuam na linha de frente enfrentam todo tipo de pressão, incluindo sobrecarga de trabalho, inexistência de pausas, cuidado de pacientes ansiosos, depressivos e com risco de complicações e morte, o que propicia quadros variados de sofrimento com efeitos duradouros na saúde do indivíduo ${ }^{7-9}$.

No presente estudo, observou-se que uma parcela dos residentes apresentou baixo senso de RP. Estudo realizado com 60 residentes multiprofissionais identificou associação significativa entre senso de RP e as seguintes variáveis: mudanças nos setores de atuação, interrupção das atividades teóricas, diminuição de atendimentos em suas especialidades e supervisão inadequada. Além desses fatores afetarem a RP, contribuem para afastamento do curso e depressão ${ }^{11}$. Por outro lado, altos escores em RP, podem ter papel protetor frente ao risco de Síndrome de Burnout e transtornos mentais comuns, estando relacionados às recompensas percebidas pelo residente quanto ao respeito, tratamento justo, suporte por parte da preceptoria e de colegas de trabalho em momentos difíceis diminuindo os efeitos dos estressores psicossociais ${ }^{15,22-24}$.

Identificou-se no estudo (Tabela 1) que os residentes foram afastados do curso devido à infecção pelo Covid-19 e outros problemas de saúde, sendo que uma parte do grupo confirmou acompanhamento pela saúde mental. Salientase que o suporte psicossocial é relevante no enfrentamento dos estressores, tendo a instituição, gestores e preceptores papel relevante no que diz respeito a estruturação do curso, supervisão das práticas e acolhimento, pois ao se colocarem como rede de apoio psicossocial junto especializandos têm muito a contribuir na minimização dos medos e/ou inseguranças diante de uma doença nova, letal e de forte impacto psicossocial ${ }^{10-12}$. 
Assim, medidas de controle do estresse ocupacional são cruciais para a proteção da saúde mental do residente durante a pandemia e entre elas, oferta de apoio psicológico, redução da jornada de trabalho, valorização profissional, melhoria nas condições de trabalho e apoio social ${ }^{25}$. Portanto, o suporte institucional é inquestionável na adoção de medidas protetivas a saúde dos residentes e na adoção de estratégias de enfrentamento saudáveis, principalmente ao se observar que parte significativa dos residentes afirmou o consumo de bebidas alcóolicas.

Sobre o consumo de álcool pela população geral, estudo multicêntrico ( $n=12.328$ ) em 33 países da América Latina evidenciou o comportamento de beber socialmente online em maior frequência e o consumo pesado como estratégia defensiva em resposta ao isolamento social e a sintomas de ansiedade ${ }^{26}$.

\section{CONCLUSÃO}

A amostra foi composta majoritariamente por residentes do sexo feminino, casados e maiores de 25 anos. Uma parcela dos residentes preencheu os critérios para burnout com risco de desenvolvimento da síndrome, devido a altos escores na subescalas exaustão emocional, despersonalização e baixo senso de realização profissional. Apesar de não terem sido realizados testes estatísticos, no intuito de estabelecer a associação entre as variáveis e burnout, observouse que algumas características como sexo feminino, estar no segundo ano da residência, não ter experiência profissional, ter se afastado para tratamento médico e inclusive devido a Covid-19, podem ter contribuído para altos escores nas subescalas exaustão emocional, despersonalização acompanhados de baixa realização profissional.

A opção metodológica, o número de participantes e os poucos estudos sobre burnout em residentes durante a pandemia são fatores limitantes em termos de discussão dos resultados e generalização para outros contextos de formação. Os dados apresentados são relevantes por ratificar o papel das instituições formadoras, gestores e preceptores na prevenção do estresse psicossocial mediante suporte social e técnico do grupo, provisão de pessoal e insumos materiais que garantam a realização de práticas seguras nas unidades de tratamento de Covid-19 e com menor risco de exposição e adoecimento.

O risco de Burnout requer ainda o reconhecimento pelos próprios residentes dos sintomas preditores que podem servir de alerta para o seu desenvolvimento, pois a não identificação dos sintomas e procura por suporte em saúde mental pode conduzir o residente a adotar mecanismos de enfrentamento pouco eficazes como o uso de bebidas alcoólicas, faltas e até mesmo abandono do curso. Por se tratar de mecanismos adaptativos individuais, cabe ao Curso de Residência proporcionar ações preventivas de cunho coletivo com o intuito de fortalecer a formação profissional e promover a saúde do grupo.

\section{REFERÊNCIAS}

1. World Health Organization [site da internet]. WHO Coronavirus (COVID-19) Dashboard. 2021 [cited 2021 Jun 11]. Available from: https://covid19.who.int.

2. World Health Organization. Coronavirus disease (COVID-19) outbreak: rights, roles and responsibilities of health workers, including key considerations for occupational safety and health: interim guidance. 2020. World Health Organization. [cited 2021 Jun 11]. Available from: https://apps.who.int/iris/handle/10665/331510.

3. Sant'Ana G, Imoto AM, Amorim FF, Taminato M, Peccin MS, Santana LA, et al. Infection and death in healthcare workers due to COVID-19: a systematic review. Acta Paul. Enferm. [Internet]. 2020 [cited 2021 Jun 16]; 33(eAPE20200107):1-9. DOI: https://doi.org/10.37689/acta-ape/2020A00107.

4. Barbosa DJ, Gomes MP, Souza FBA, Gomes AMT. Stress factors in nursing professionals in combating the COVID-19 pandemic: synthesis of evidence. Comun. Ciênc. Saúde [Internet]. 2020 [cited 2021 Apr 19]; 31(Suppl 1):31-47. Available from: http://www.escs.edu.br/revistaccs/index.php/comunicacaoemcienciasdasaude/article/view/651.

5. Souza NVDO, Carvalho EC, Soares SSS, Varella TCMML, Pereira SRM, Andrade KBS. Nursing work in the COVID-19 pandemic and repercussions for workers' mental health. Rev. Gaúch. Enferm. [Internet]. 2021 [cited 2021 Jul 05]; 42(e20200225):1-6. DOI: https://doi.org/10.1590/1983-1447.2021.20200225.

6. Yang Y, Li W, Zhang Q, Zhang L, Cheung T, Xiang YT. Mental health services for older adults in China during the COVID-19 outbreak. Lancet Psychiatry [Internet]. 2020 [cited 2021 Apr 19]; 7(4):e19. DOI: https://doi.org/10.1016/S2215-0366(20)30079-1.

7. Spoorthy MS, Pratapa SK, Mahant S. Mental health problems faced by healthcare workers due to the COVID-19 pandemic-a review. Asian J. Psychiatr. [Internet]. 2020 [cited 2021 Apr 19]; 51:102119. DOI: https://doi.org/10.1016/j.ajp.2020.102119.

8. Cai H, Tu B, Ma J, Chen L, Fu L, Jiang Y, et al. Psychological Impact and Coping Strategies of Frontline Medical Staff in Hunan Between January and March 2020 During the Outbreak of Coronavirus Disease 2019 (COVID-19) in Hubei, China. Med. Sci. Monit. [Internet]. 2020 [cited 2021 Apr 18]; 26:e924171. DOI: https://doi.org/10.12659/MSM.924171.

9. Li S, Wang Y, Xue J, Zhao N, Zhu T. The Impact of COVID-19 Epidemic Declaration on Psychological Consequences: A Study on Active Weibo Users. Int. J. Environ. Res. Public Health [Internet]. 2020 [cited 2021 Apr 18]; 17(6):2032. DOI: https://doi.org/10.3390/ijerph17062032. 
10. Borges FES, Aragão DFB, Borges FES, Borges FES, Sousa ASJ, Machado ALG. Risk factors for burnout syndrome in health professionals during the covid-19 pandemic. Enferm. Atual. [Internet]. 2021 [cited 2021 Jun 16]; 95(33):e-21006. DOI: https://doi.org/10.31011/reaid-2020-v.94-n.32-art.835.

11. Moreira APF, Patrizzi LJ, Accioly MF, Shimano SGN, Walsh IAP. Quality of life evaluation, sleep and Burnout Syndrome the residents of the multidisciplinary residency program in health. Medicina [Internet]. 2016 [cited 2021 Jul 13]; 49(5):393-402. DOI: https://doi.org/10.11606/issn.2176-7262.v49i5p393-402.

12. Cavalcanti IL, Lima FLT, Souza TA, Silva MJS. Burnout and depression in residents of a Multi-professional Program in Oncology: a longitudinal prospective study. Rev. Bras. Educ. Méd. [Internet]. 2018 [cited 2021 Apr 18]; 42(1):190-8. DOI: https://doi.org/10.1590/1981-52712018v42n1RB20170078.

13. Valério RL, Oliveira EB, Kestenberg CCF, Paula VG, Dias LBS, Oliveira TS. Emotional exhaustion in resident nurses of specialized units in a university hospital. Res. Soc. Dev. [Internet]. 2020 [cited 2021 May 05]; 9(2):e198922240. DOI: http://dx.doi.org/10.33448/rsd-v9i2.2240.

14. Bond MMK, Oliveira MS, Bressan BJ, Bond MMK, Silva ALFA, Merlo ARC. Prevalence of Burnout among Medical Residents of a University Hospital. Rev. Bras. Educ. Méd. [Internet]. 2018 [cited 2021 Apr 18]; 42(3): 97-107. DOI: https://doi.org/10.1590/1981-52712015v42n3RB20170034.r3.

15. Oliveira EB, Carvalho RAC, Teixeira E, Zeitoune RCG, Sabóia VM, Gallasch CH. Factors involved in the training of resident nurses: view of alumni from a residency program. Rev. Min. Enferm. [Internet]. 2017 [cited 2021 May 05]; 21:e-1064. DOI: https://doi.org/10.5935/1415-2762.20170074.

16. Silva DFO, Cobucci RN, Soares-Rachetti VP, Lima SCVC, Andrade FB. Prevalence of anxiety among health professionals in times of COVID-19: a systematic review with meta-analysis. Ciênc. Saúde Colet. [Internet]. 2021 [cited 2021 Jun 26]; 26(02):693-710. DOI: https://doi.org/10.1590/1413-81232021262.38732020.

17. Maslach C, Leiter MP, Jackson SE. Making a significant difference with burnout interventions: researcher and practitioner collaboration. J. Organ. Behav. [Internet]. 2012 [cited 2021 Jun 15]; 33:296-300. DOI: https://doi.org/10.1002/job.784.

18. Benevides-Pereira AMT. Burnout: quando o trabalho ameaça o bem-estar do trabalhador. São Paulo: Casa do Psicólogo, 2014.

19. Lautert L. The profesional fatigue: empirical study with hospital nurses. Rev. Gaúch. Enferm. [Internet]. 1977 [cited 2021 May 05]; 18(2):133-44. Available from: https://seer.ufrgs.br/RevistaGauchadeEnfermagem/article/view/4140.

20. Conselho Federal de Enfermagem. Resolução Cofen no 657/2020. Estabelece os requisitos mínimos para o registro de enfermeiro especialista, na Modalidade de Residência em Enfermagem. Brasília (DF): Diário Oficial da União. 2014. [cited 2021 Apr 15]. Available from: http://www.cofen.gov.br/resolucao-cofen-no-04592014_26170.html.

21. Ministério da Saúde (Br). Conselho Nacional de Saúde. Resolução no 466, de 12 de dezembro de 2012. Dispõe sobre diretrizes e normas regulamentadoras de pesquisas envolvendo seres humanos. Brasília (DF): Diário Oficial da República Federativa do Brasil, 2013. [cited 2021 Oct 18]. Available from: https://bvsms.saude.gov.br/bvs/saudelegis/cns/2013/res0466_12_12_2012.html.

22. Menegatti MS, Rossaneis MA, Schneider P, Silva LGC, Costa RG, Haddad MCFL. Stress and coping strategies used by nursing interns. Rev Min Enferm [Internet]. 2020 [cited 2021 Jun 16]; 24:e1329. DOI: https://doi.org/10.5935/1415-2762.20200066.

23. Freitas MA, Silva Junior OC, Machado DA. Stress level and quality of life of resident nurses. Rev. Enferm. UFPE on line [Internet]. 2016 [cited 2021 Jun 16]; 10(2):623-30. Available from: https://periodicos.ufpe.br/revistas/revistaenfermagem/article/view/10998.

24. Falco CB, Fabri JMG, Oliveira EB, Silva AV, Faria MGA, Kestenberg CCF. Mental disorders common among nursing residents: an analysis based on the Self-Reporting Questionnaire. Rev. enferm. UERJ [Internet]. 2019 [cited 2021 Jun 16]; 27:e39165. DOI: https://doi.org/10.12957/reuerj.2019.39165.

25. Helioterio MC, Lopes FQRS, Sousa CC, Souza FO, Pinho PS, Sousa FNF, et al. COVID-19: why the protection of health workers is a priority in the fight against the pandemic? Trab. educ. saúde [Internet]. 2020 [cited 2021 Jun 10]; 18(3):e00289121. DOI: https://doi.org/10.1590/1981-7746-sol00289.

26. Garcia-Cerde R, Valente JY, Sohi I, Falade R, Sanchez ZM, Monteiro MG. Alcohol use during the COVID-19 pandemic in Latin America and the Caribbean. Rev. Panam. Salud Publica [Internet]. 2021 [cited 2021 Jun 25]; 45:e52. DOI: https://doi.org/10.26633/RPSP.2021.52. 